\title{
A novel histone H3K4me1-specific binding protein is required for siRNA accumulation and DNA methylation at a subset of loci targeted by RNA- directed DNA methylation
}

\section{Qingfeng Niu}

Shanghai Center for Plant Stress Biology, and National Key Laboratory of Plant Molecular Genetics, Center of Excellence in Molecular Plant Sciences, Chinese Academy of Sciences

\section{Zhe Song}

Shanghai Center for Plant Stress Biology, and National Key Laboratory of Plant Molecular Genetics, Center of Excellence in Molecular Plant Sciences, Chinese Academy of Sciences https://orcid.org/00000002-3823-0261

\section{Kai Tang}

https://orcid.org/0000-0003-3913-6426

\section{Lixian Chen}

Shanghai Center for Plant Stress Biology, and National Key Laboratory of Plant Molecular Genetics, Center of Excellence in Molecular Plant Sciences, Chinese Academy of Sciences

\section{Ting Ban}

Shanghai Center for Plant Stress Biology, and National Key Laboratory of Plant Molecular Genetics, Center of Excellence in Molecular Plant Sciences, Chinese Academy of Sciences

\section{Zhongxin Guo}

Shanghai Center for Plant Stress Biology, and National Key Laboratory of Plant Molecular Genetics, Center of Excellence in Molecular Plant Sciences, Chinese Academy of Sciences

\section{Chanhong Kim}

Chinese Academy of Sciences https://orcid.org/0000-0003-4133-9070

\section{Heng Zhang}

Shanghai Center for Plant Stress Biology, and National Key Laboratory of Plant Molecular Genetics, Center of Excellence in Molecular Plant Sciences, Chinese Academy of Sciences

\section{Cheng-Guo Duan}

Shanghai Center for Plant Stress Biology, Chinese Academy of Sciences https://orcid.org/0000-00030527-5866

\section{Huiming Zhang}

Chinese Academy of Sciences https://orcid.org/0000-0003-0695-3593

\section{Jian-Kang Zhu}


Shanghai Center for Plant Stress Biology and Center for Excellence in Molecular Plant Sciences, Chinese Academy of Sciences

\section{Jiamu Du}

Southern University of Science and Technology https://orcid.org/0000-0002-1337-0786

\section{Zhaobo Lang ( $\nabla$ zblang@psc.ac.cn )}

Shanghai Center for Plant Stress Biology, and National Key Laboratory of Plant Molecular Genetics, Center of Excellence in Molecular Plant Sciences, Chinese Academy of Sciences

\section{Article}

Keywords: DNA methylation, siRNA, histone modification, RdDM

Posted Date: November 4th, 2020

DOl: https://doi.org/10.21203/rs.3.rs-98860/v1

License: (c) (i) This work is licensed under a Creative Commons Attribution 4.0 International License. Read Full License

Version of Record: A version of this preprint was published at Nature Communications on June 7th, 2021. See the published version at https://doi.org/10.1038/s41467-021-23637-4. 


\section{Abstract}

In plants, RNA-directed DNA methylation (RdDM) is a well-known de novo DNA methylation pathway that involves two plant-specific RNA polymerases, Pol IV and Pol V. In this study, we discovered and characterized a new RdDM factor, RDM15. Through DNA methylome and genome wide siRNA analyses, we show that RDM15 is required for RdDM-dependent DNA methylation and siRNA accumulation at a subset of RdDM target loci. We show that RDM15 contributes to Pol V-dependent downstream siRNA accumulation, and interacts with NRPE3B, a subunit specific to Pol V. We also show that the C-terminal tudor domain of RDM15 specifically recognizes the histone 3 lysine 4 monomethylation (H3K4me1) mark. Structure analysis of RDM15 in complex with the H3K4me1 peptide showed that the RDM15 tudor domain specifically recognizes the monomethyllysine through an aromatic cage and a specific hydrogen bonding network; this chemical feature-based recognition mechanism differs from all previously reported lower methyllysine recognition mechanisms. RDM15 and H3K4me1 have similar genome-wide distribution patterns at RDM15-dependent RdDM target loci, establishing a link between H3K4me1 and RDM15-mediated RdDM in vivo. In summary, we have identified and characterized a histone H3K4me1specific binding protein as a new RdDM component, and our structural analysis of RDM15 revealed a new type of chemical feature-based lower methyllysine recognition mechanism.

\section{Introduction}

DNA methylation mainly refers to an addition of a methyl group to the fifth position of cytosine, resulting in 5'-methylcytosine (5-mC). Such methylation is involved in gene imprinting, fruit ripening, maintenance of genome integrity, and other important biological functions ${ }^{1}$. In mammals, DNA methylation mainly occurs in the CG context; but can be found in all three sequence contexts, including CG, CHG, and CHH (H $=\mathrm{T}, \mathrm{A}$, or $\mathrm{C}$ ) in plants ${ }^{2}$. Cytosines in different sequence contexts can be methylated by different methyltransferases: cytosine in all sequence contexts can be de novo methylated by DRM2 (DOMAINS REARRANGED METHYLTRANSFERASE 2) through the RNA-dependent DNA methylation (RdDM) pathway; CG and CHG methylation is maintained by MET1 (METHYLTRANSFERASE 1) and CMT3 (CHROMOMETHYLASE 3), respectively, while CHH methylation is maintained by CMT2 or DRM2, depending on the chromatin context ${ }^{1,3-7}$

The RdDM pathway is responsible for DNA methylation and transcriptional silencing of transposons and other repetitive elements, and is critical for pathogen defense, stress response, and many other processes in plants ${ }^{1,8,9}$. This pathway comprises two main steps that depend on the plant-specific RNA polymerases Pol IV and Pol V. The first step is Pol IV-dependent siRNA biogenesis that involves not only Pol IV but also several other RdDM factors, such as RDR2 (RNA-DEPENDENT RNA POLYMERASE 2) and DCL3 (DICER-LIKE 3). The second step is siRNA-guided DNA methylation, in which Pol V-transcribed longnoncoding RNA serves as a scaffold RNA, and DRM2 is recruited to catalyze DNA methylation. This recruitment of DRM2 involves base-pairing between AGO4 (ARGONAUTE 4)- and AGO6-bound siRNAs 
and the scaffold RNA and the participation of RdDM factors such as KTF1 (KOW DOMAIN-CONTAINING TRANSCRIPTION FACTOR 1), DRD1, DMS3 and RDM1 (RNA-DIRECTED DNA METHYLATION 1) $9,10$.

Different epigenetic marks, such as DNA methylation and histone modifications, can interact to regulate the chromatin state and DNA methylation level in the genome ${ }^{11}$. Histone modifications are established by histone modification enzymes known as writer proteins, and can be recognized by reader proteins to regulate downstream effector molecules. To date, with few exceptions, all of the reported recognition mechanisms of histone mark readers are conserved between plants and animals ${ }^{12,13}$.

In RdDM, the recruitment of Pol IV requires SHH1 (SAWADEE HOMEODOMAIN HOMOLOG 1), which possesses an SAWADEE domain that can bind to both unmethylated H3K4 and methylated H3K9 ${ }^{14,15}$. At RdDM target loci, active histone marks, such as H3K4me2 and H3K4me3, function to prevent RdDM ${ }^{16}$. On the other hand, the recruitment of Pol V requires the participation of SUVH2 and SUVH9 17, 18. Although these two proteins lack histone methyltransferase activities, they both have an SRA domain that can bind to methylated DNA ${ }^{18,19}$, indicating that they contribute to the interplay between Pol V and chromatin by binding to pre-existing methylated DNA. The crosstalk between histone modification and RdDM has been extensively studied, but the existing model cannot explain all of the targeting mechanisms of DNA methylation. Additional studies are required to increase our understanding of the interaction between epigenetic modification and RdDM components.

In the current research, we found a new chromatin-binding protein, RDM15, that is involved in RdDM. Through whole-genome bisulfite sequencing and siRNA sequencing of rdm 15 mutants and the wild type (WT), we demonstrate that RDM15 is required for RdDM-dependent DNA methylation and RdDMdependent siRNA accumulation at a subset of RdDM target regions. In addition, protein structure analysis revealed that RDM15 can specifically recognize H3K4me1 through its C-terminal tudor domain. The RDM15 tudor domain uses an aromatic cage and a hydrogen bonding network to achieve the monomethyllysine-specific recognition of H3K4me1; this chemical feature-based lower methylation statespecific readout mechanism has not been discovered previously. Our results also show RDM15 protein enrichment at RDM15-dependent RdDM target regions and physical interaction between RDM15 and a PolV subunit. In summary, our study has identified a novel RdDM component, RDM15, and has elucidated the molecular mechanism underlying RDM15 function in the RdDM pathway.

\section{Results}

\section{RDM15 is required for transcriptional silencing at RdDM target loci}

To clone the RDM15 gene, we used TAIL-PCR and found a T-DNA insertion in the $7^{\text {th }}$ exon of AT4G31880 (Fig. 1c). A complementation test showed that a transgene containing the native promoter-driven genomic sequence of AT4G31880 can rescue the pRD29A-LUC silencing phenotype of the rdm15-1/ros 11 double mutant (Supplementary Fig. 1c). To confirm the silencing function of RDM15, we ordered two T-DNA insertion alleles of RDM15 (Col-0 background): Salk_013481 and Salk_024055 (hereafter referred 
to as $r d m 15-2$ and $r d m 15-3)$. Both of these mutants contain T-DNA insertions in the $7^{\text {th }}$ exon of RDM15 (Fig. 1c and Supplementary Fig. 1d). The silencing of AtGP1 and AtMEA-ISR, two known RdDM targets ${ }^{23}$, is released by both $r d m 15-2$ and $r d m 15-3$ mutations (Fig. 1d), supporting the notion that RDM15 is required for the silencing of RdDM target loci.

RdDM controls the expression of the DNA demethylase ROS1 via methylation of the MEMS sequence located in the promoter region of $R O S 1^{24,25}$. ROS1 expression is dramatically decreased in many RdDM mutants, including $n r p d 1 a, n r p d 1 b$, and $a g o 4^{24,25}$. We found that $r d m 15$ mutants have lower ROS1 transcript levels (Fig. 1e and Supplementary Fig. 1e), although the levels are not as low as in nrpd or ago4 mutants. These data suggest that RDM15 functions in RdDM-dependent transcriptional gene silencing (TGS).

\section{RDM15 is required for RdDM-dependent DNA methylation}

To characterize the effect of RDM15 on DNA methylation, we generated single-base resolution maps of DNA methylation for Col-0, rdm15-2, and rdm15-3, with two biological replicates for each mutant. Principal components analysis showed very good consistency between the replicates (Supplementary Fig. 2a). Using the R package methylKit ${ }^{26}$, which considers variations among replicates of two mutant alleles, we identified 1,390 differentially methylated regions (DMRs) in rdm15 mutants compared with the WT; most of the DMRs (1,354 out of 1,390) were hypomethylated, suggesting that RDM15 is required for DNA methylation at more than 1,300 endogenous genomic regions (Supplementary Table 1). We compared the DNA methylation patterns of $r d m 15$ mutants with those of known RdDM mutants. NRPD1 and NRPE1 are the largest subunits exclusive to Pol IV and Pol V, respectively. Using the same methylKit method and compared to the WT, we identified 4,293 hypo DMRs in nrpd1-3 (Col-0 ecotype) and 4,629 hypo DMRs in nrpe1-11 (Col-0 ecotype) (Supplementary Table 1). We found that the Pol IV, Pol V, and RDM15 targets have comparable genomic compositions. Among the hypo DMRs, $82 \%$ in $n r p d 1-3,81 \%$ in nrpe1-11, and 79\% in rdm15 are in TE regions (Fig. 2a). Similar to known RdDM targets, rdm15 DMRs show DNA hypomethylation in all three sequence contexts ( $\mathrm{mCG}, \mathrm{mCHG}$, and $\mathrm{mCHH}$; Fig. $2 \mathrm{~b}$ ), although the methylation level in $r d m 15$ mutants is not as low as in nrpd1-3. We further analyzed the overlap between RDM15 targets and known RdDM targets. As shown in Fig. 2c, 94\% $(1,269 / 1,354)$ and 95\% $(1,281 / 1,354)$ of $r d m 15$ hypo DMRs overlap with the $n r p d 1$ and $n r p e 1$ hypo DMRs, respectively. DNA methylation profiles of several representative $r d m 15$ hypo DMRs are shown in Fig. $2 \mathrm{~d}$ and Supplementary Fig. 2b. These results revealed that RDM15 is required for methylation at a subset of RdDM target loci, suggesting that RDM15 may be a novel RdDM component. 
We generated genome-wide siRNA profiles for WT (Col-0), rdm15-2, and rdm15-3 with two biological replicates for each genotype (Supplementary Fig. 3a). In Arabidopsis, RdDM has two main steps, i.e., biogenesis of 24-nt siRNAs and guidance of DRM2 to RdDM target by the siRNAs. Genomic regions showing RdDM-dependent DNA methylation always have siRNA enrichment ${ }^{15}$. To determine whether RDM15 is involved in siRNA biogenesis, we compared rdm15 mutants with Col-0, and identified 2,808 RDM15-dependent siRNA clusters (hereafter referred to as RDM15 siRNAs) (Supplementary Table 2) that showed decreased 24-nt siRNA accumulation in rdm15 mutants relative to Col-0. This result suggested that RDM15 is required for 24nt siRNA accumulation in vivo (Fig. 3a).

RdDM-dependent siRNAs can be classified into upstream siRNAs (siRNAs dependent on Pol IV only) and downstream siRNAs (siRNAs dependent on both Pol IV and Pol V) ${ }^{15}$.The upstream siRNAs are affected only in mutants defective in upstream RdDM components, such as $n r p d 1$, whereas the downstream siRNAs are not only affected in these mutants but also in mutants defective in downstream RdDM components, such as nrpe 1 and drm2 (Fig. 3b). To position RDM15 in the RdDM pathway, we examined the enrichment of RDM15-dependent siRNAs, and found that RDM15 siRNA levels are lower in nrpd1-4, nrpe1-12, and drm2-2 compared to the WT (Fig. 3b). In addition, the Pol IV-only siRNAs are not significantly affected in $r d m 15$ mutants (Fig. 3a). These results showed that RDM15 is mainly required for the accumulation of downstream siRNAs in the RdDM pathway, suggesting that RDM15 functions in a downstream step of RdDM.

To further understand the relationship between the siRNAs and DNA methylation in rdm15 mutants, we monitored changes in siRNA enrichment at hypo DMRs in rdm15 mutants. The siRNA levels were clearly lower at hypo-DMRs in rdm15 mutants than in the WT (Fig. 3c). Consistent with this result, the DNA methylation level, including $\mathrm{mCG}, \mathrm{mCHG}$, and $\mathrm{mCHH}$, was decreased in regions of RDM15-dependent siRNA clusters (Fig. 3d and Supplementary Fig. 3b). The siRNA levels at several representative hypoDMRs are shown in Fig. 3e and Supplementary Fig.3c. These results suggest that RDM15 influences DNA methylation at RdDM target regions by regulating siRNA accumulation.

\section{RDM15 physically interacts with NRPE3B, a subunit exclusive to Pol V}

To further investigate how RDM15 affects RdDM, we identified RDM15-interacting proteins by performing immunoprecipitation (IP) followed by liquid chromatography-tandem mass spectrometry (LC-MS/MS) with RDM15-3xMYC/rdm15-3 and RDM15-3xFLAG/rdm15-3 plants (Supplementary Fig. 4). WT plants that do not express RDM15-3xMYC or RDM15-3xFLAG were used as controls. We found that NRPE3B, a subunit exclusive to Pol V, was co-immunoprecipitated with anti-MYC antibodies and anti-FLAG antibodies in the RDM15-3XMYC and RDM15-3xFLAG transgenic plants, respectively, but not in the 
control plants (Fig. 4a and Supplementary Table 3). We validated the interaction between RDM15 and NRPE3B using a split luciferase complementation assay and a BiFC assay in tobacco leaves (Fig. $4 \mathrm{~b}$ and 4c). These data indicated that RDM15 may affect RdDM by interacting with Pol V.

\section{The RDM15 tudor domain specifically recognizes H3K4me1}

The results above show that RDM15 functions in the regulation of siRNA accumulation and DNA methylation at a subset of RdDM target loci. To further investigate how RDM15 functions, we analyzed its protein sequence and found two domains with known functions: a N-terminal ARM repeat, which is known to mediate protein-protein interactions, and a C-terminal tudor domain, which functions as a histone mark reader (Fig. 5a). The tudor domain has been found in many proteins related to epigenetic regulation and acts as a histone mark reader module that recognizes methylated lysine or arginine marks

27, 28 . In plants, histone mark readers sometimes have plant-specific binding targets and functions ${ }^{12}$. This prompted us to explore the histone mark binding property of the RDM15 tudor domain. In our assay using histone peptide arrays, which contained several hundred combinations of histone marks, the RDM15 tudor domain showed a significant binding to H3K4me1 and H4K20me1 marks (Supplementary Fig. 5a and Supplementary Table 4). We used isothermal titration calorimetry (ITC) to further confirm the binding and to measure the binding affinity between the RDM15 tudor domain and different methyllysinemodified histone peptides. The H3K4me1 peptide yielded the strongest binding (1.47 mM) (Fig. 5b), while the H4K20me1 yielded a 15-fold lower binding affinity (22.9 mM) (Supplementary Fig. 5b), indicating that H4K20me1 is not the optimal binding partner for RDM15. In addition, researchers previously reported that the existence of H4K20 methylation in plants is controversial and that no in vivo H4K20 methylation could be detected by mass spectrometry ${ }^{29}$. We therefore focused on the interaction between the H3K4me1 mark and RDM15. We assessed the binding between the RDM15 tudor domain and peptides with different H3K4 methylation states. ITC measurements clearly showed that RDM15 tudor domain bound much more strongly to the monomethylation state of H3K4 than to the unmethylated H3K4 (H3K4me0) or to the higher methylation states of H3K4 (H3K4me2 and H3K4me3) (Fig. 5b).

\section{The structure of RDM15 tudor domain in complex with H3K4me1 peptide}

To further investigate the molecular mechanism underlying the specific recognition of the H3K4me1 mark by RDM15, we carried out structural studies. The crystal structure of the RDM15 tudor domain in complex with the $\mathrm{H} 3(1-15) \mathrm{K} 4 \mathrm{me} 1$ peptide was determined using the SAD method and was refined to $1.7-\AA$ resolution (Supplementary Table 5, Fig. 5c). Although a 15-residue H3K4me1 peptide was used in the crystallization, only the H3A1 to H3Q5 segment of the peptide exhibited well-defined electron density and was built into the final model (Fig. 5c). The tudor domain of RDM15 exhibits a canonical tudor domain fold with five $b$-strands forming a twisted $b$-barrel structure that resembles the structure of other tudor domains (Fig. 5c) 27. 
Our results show that the RDM15 tudor domain can specifically recognize H3K4me1. The main chain of the H3K4me1 peptide has a ' $\mathrm{U}$ '-shaped conformation such that the N-terminal H3A1 and the C-terminal H3Q5 extend out away from the peptide-protein binding interface (Fig. 5c). The side chains of H3R2 and H3K4me1 form a pincer-like conformation, thereby anchoring on two adjacent negatively charged surface pockets of the tudor domain and highlighting a significant structural and chemical complementarity (Fig. 5d). In detail, the H3R2 inserts its side chain into a negatively charged pocket and forms hydrogenbonding interactions with Glu647 and Gln654 of RDM15, as well as a salt bridge interaction with Glu647 (Fig. 6a). For H3K4me1, the interactions can be divided into two parts. Three aromatic residues, Trp616, Tyr623, and Tyr641, form an aromatic cage to accommodate the methyl group of the monomethyllysine from one side, which is similar to other canonical methyllysine readers ${ }^{28,30}$ (Fig. 6b). On the other side, the two free protons of the monomethylammonium group are fully coordinated with Asp643 and Asp645 by hydrogen bonding and salt bridge interactions (Fig. 6b), representing a new monomethyllysine recognition mechanism (Supplementary Fig. 6 and see detailed analysis in the Discussion). The H3T3 positioned between H3R2 and H3K4me1 stretches its side chain against the protein and is not involved in the interaction with the RDM15 tudor domain (Fig. 5c). In general, both H3R2 and H3K4me1 are specifically recognized by a hydrogen bonding network and electrostatic interactions involving their charged side chains, as well as by an aromatic cage that captures the methylation modification (Fig. $6 \mathrm{c}$ ). Mutations of residues of either the aromatic cage or of those involved in hydrogen bonding interactions with the peptide result in a significant decrease in the binding affinity (Fig. 6d and 6e), revealing that these residues are critical for the recognition of H3K4me1 by RDM15.

In the structure of the RDM15-H3K4me1 complex, the recognition of the H3 tail by the RDM15 tudor domain highlights the specific recognition of H3R2 and H3K4me1, while other surrounding residues and the intervening H3T3 do not contribute to the recognition. The RDM15 tudor domain therefore recognizes an $\underline{R} X \underline{K m e 1}$ (X here stands for any residue) motif on histone tails. Among all four types of Arabidopsis histone proteins, there are two additional sites that contain the same motif and that might be recognized by the RDM15 tudor domain: $\mathrm{H} 2 \mathrm{~A} \underline{\mathrm{R} 3} \mathrm{TK} 5$ and $\mathrm{H} 4 \underline{\mathrm{R} 3 \mathrm{GK}} \underline{\mathrm{K}}$. A previous mass spectrometry study showed that the lysine residues of both of these two sites can be acetylated, but no methylation modification was

identified in vivo ${ }^{29}$. It follows that although the RDM15 tudor domain only recognizes two residues on the histone tail, the specific RXKme1 pattern only occurs in the H3K4 region, which ensures that recognition only occurs at H3K4me1, thereby explaining the sequence specificity.

\section{Characterization of chromatin targets of RDM15 binding}

We performed chromatin immunoprecipitation using native promoter-driven RDM15-3xFLAG/rdm15-3 followed by high throughput sequencing (ChIP-seq). We observed higher RDM15 protein enrichment in 
RDM15-dependent siRNA regions than in Pol IV-only siRNA regions (Fig. 7a), which is consistent with our above finding that RDM15 is required for the accumulation of RDM15 siRNAs but does not affect the accumulation of Pol IV-only siRNAs (Fig. 3). When we ranked RDM15 siRNA regions by the difference in siRNA accumulation between $r d m 15$ and the WT, we found that the change in siRNA accumulation ( $r d m 15$ vs. the WT) was positively correlated with RDM15 protein enrichment; the change in siRNA accumulation in $n r p d 1$ vs. the WT served as the negative control (Fig. 7b and Supplementary Fig. 7a). These results suggest that the influence of RDM15 on siRNA accumulation is correlated with its protein enrichment. In addition, the distribution pattern of $\mathrm{H} 3 \mathrm{~K} 4 \mathrm{me} 1$ but not of $\mathrm{H} 3 \mathrm{~K} 4 \mathrm{me} 2$ and $\mathrm{H} 3 \mathrm{~K} 4 \mathrm{me} 3$ was similar to that of RDM15 (Fig. 7c and Supplementary Fig. 7b), which is consistent with our finding of high affinity binding of RDM15 to H3K4me1. The enrichment of RDM15 and H3K4me1 is shown for several representative RDM15 target regions in Fig. 7d and Supplementary Fig. 7c.

\section{Discussion}

RdDM is an important DNA methylation pathway in plants and has been extensively studied during the past two decades. Several forward and reverse genetics screens, including the ros 1 suppressor screen in this study, were used to identify RdDM components ${ }^{20,31-34}$. Through this ros 1 suppressor screen, several novel RdDM mutants, such as $r d m 1, r d m 4$, and $k t f 1$, were identified ${ }^{21-23}$. In the current study, a new RdDM mutant, $r d m 15$, was isolated and characterized. The methylome and siRNA analyses revealed that RDM15 is required for RdDM-dependent siRNA accumulation and DNA methylation at a subset of RdDM targets. In addition, the siRNA analysis and the interaction of RDM15 with Pol V suggested that RDM15 functions in the downstream steps of the RdDM pathway.

Several histone modifications, such as $\mathrm{H} 3 \mathrm{~K} 9 \mathrm{me} 2$, have been reported to be associated with RdDM targets

${ }^{11}$. Here, we showed an association of H3K4me1 with RDM15-dependent RdDM targets. Structural analysis demonstrated that RDM15 can specifically recognize and bind to H3K4me1 (Fig. 5). In addition, ChIP-seq results showed that the genomic distribution of RDM15 protein enrichment is similar to that of H3K4me1 but not H3K4me2/3 (Fig. 7 and Supplementary Fig. 7). H3K4me1 is usually thought to be associated with actively transcribed genes ${ }^{35,36}$. Among heterochromatic regions, Pol V-transcribed regions are presumably more active than untranscribed regions. We speculate that RDM15 may help recruit Pol V to a subset of RdDM targets by recognizing the H3K4me1 mark at these genomic regions.

Many structural and functional studies have identified the general principles explaining the specific recognition of histone lysine methylation marks ${ }^{28,30}$. However, only a few studies have investigated the specific recognition of lower methyllysine, especially the monomethyllysine. L3MBTL1, a previously reported lower methylated lysine-specific binding protein, can specifically recognize mono- and dimethyllysine via the narrow and deep surface cavity of the protein (Supplementary Fig. 6a) ${ }^{37,38}$. In the recognition mode of $L 3 M B T L 1$, almost the entire side chain of the methyllysine inserts into a deep cavity on the L3BMTL1 protein surface (Supplementary Fig. 6a). The size of the cavity therefore limits the size of the substrate. For L3MBLT1, the methyllysine must be in a lower methylation states of di- or 
monomethyllysine, representing a physical selection mechanism. The molecular mechanism for the specific recognition of $\mathrm{H} 3 \mathrm{~K} 4 \mathrm{me} 1$ by the $\mathrm{CW}$ domain of Arabidopsis SDG8 was recently determined ${ }^{39}$. Like L3MBTL1, the SDG8 CW domain has a narrow cavity that accommodates the small side chain of H3K4me1 (Supplementary Fig. 6b), i.e., the recognition of H3K4me1 by SDG8 also involves physical selection ${ }^{39}$. In contrast, $\mathrm{H} 3 \mathrm{~K} 4 \mathrm{me} 1$ is anchored within a surface groove of RDM15, with only the tip of the methyl group inserting into the canonical aromatic cage (Fig. 6c). The specificity for the monomethylation of H3K4 is achieved by the full coordination of the two monomethylammonium protons by the two negatively charged residues, which increases the binding affinity for monomethylation. In other words, the specificity of H3K4me1 for RDM15 involves a chemical selection mechanism. The increase of the methylation state of the methyllysine to di- or trimethylation levels would disrupt the hydrogen bonding interaction observed between RDM15 and the H3K4me1 side chain, resulting in a decreased binding affinity as revealed by our ITC data (Fig. 5b). This selection of a lower methylation state based on chemical features clearly differs from selection based on physical shape previously reported for L3MBTL1 and SDG8, and therefore represents a new mechanism for specific monomethyllysine recognition.

The tudor domain can function in multiple modes, including single tudor, double tudor, tandem tudor, or triple-linked tudors like Spindlin1 ${ }^{27}$. A single tudor domain has been reported to be able to recognize H3K36me3, H4K20me2, and H3K4me3 ${ }^{13,27,40}$. The tudor domains of PHF1 and PHF19 have similar structures and recognition mechanisms and can specifically recognize the H3K36me3 mark; the structures of their complexes with $\mathrm{H} 3 \mathrm{~K} 36 \mathrm{me} 3$ peptide have been reported ${ }^{41-43}$, which enables us to perform a structure-based comparison. The RDM15 tudor and PHF1 tudor have a generally similar folding topology, with their aromatic cages occupying almost identical positions (Supplementary Fig. 6d) ${ }^{43}$. It is interesting that the H3K4me1 and H3K36me3 peptides occupy different positions, although their methylammonium groups occupy the same position (Supplementary Fig. 6d), suggesting that the conserved aromatic cage of the tudor domain serves as a docking site for methyllysine, with the diversified surrounding residues contributing to the histone mark specificity. PHF1 uses a four-residue aromatic cage to accommodate H3K36me3 (Supplementary Fig. 6d) ${ }^{43}$. Although Tyr47 and Phe65 of PHF1 adopt an almost identical conformation as Tyr623 and Tyr641 of RDM15, the Trp41 of PHF1 has a distinct torsion angle relative to Trp616 of RDM15, resulting in a broader pocket in PHF1, which is suitable for accommodating trimethyllysine; RDM15, in contrast, has a narrow pocket that only accommodates monomethyllysine.

Our genetic and epigenomic analyses identified RDM15 as a novel factor required for siRNA accumulation and DNA methylation at a subset of genomic regions targeted by RdDM. Our biochemical and structural results demonstrated that RDM15 is a reader of H3K4me1, and revealed a new mechanism of recognition of lower methyllsine marks. Future work will determine whether RDM15 may affect the recruitment of Pol $\mathrm{V}$ to RdDM target regions, and the mechanism of recognition of lower methyllysine mark by RDM15 may be applicable to other histone mark readers. 


\section{Materials And Methods}

\section{Plant materials and growth conditions}

The Arabidopsis C24 ecotype plant materials used in this study (WT, ros 1-1, ros 1-1 rdm15-1, ros 1-1, nrpd1) carried a homozygous T-DNA insertion that contained the p35S-NPTII and pRD29A-LUC transgenes. T-DNA mutagenized ros 1-1 populations were used for ros 1-1 suppressor screening according to luciferase signals as described previously ${ }^{21}$. Plant materials of Columbia-0 (Col) ecotype included rdm15-2 (Salk_013481), rdm15-3 (Salk_024055), nrpd1-3 (SALK_128428C), and nrpe1-11 (SALK_029919C). Plants were grown in growth rooms at $22^{\circ} \mathrm{C}$ with a $16 \mathrm{~h}-8 \mathrm{~h}$ light-dark cycle.

\section{Whole-genome bisulfite sequencing and data analyses}

Genomic DNA was extracted from 2-week-old seedlings using the Plant DNeasy mini kit (Qiagen) and was sent to the Core Facility for Genomics at the Shanghai Center for Plant Stress Biology (PSC) for whole-genome bisulfite sequencing (WGBS).

For WGBS data analysis, the raw data were trimmed using Trimmomatic ${ }^{44}$ with parameters "LEADING:20 TRAILING:20 SLIDINGWINDOW:4:15 MINLEN:50". Only clean paired-end reads were mapped to the TAIR10 genome using tool BSMAP ${ }^{45}$ with parameters "-m 0 -w 2" (other parameters were used with default values). To remove potential PCR duplicates, the "rmdup" command of SAMtools ${ }^{46}$ was used. methratio.py script from BSMAP was used to extract methylation ratios from mapping results. The $\mathrm{R}$ package methylKit ${ }^{26}$ was used to find differentially methylated regions (DMRs): the "tileMethylCounts" function was used to summarize methylated/unmethylated base counts over tilling windows (500 bp) across the genome. The "calculateDiffMeth" function was used to calculate differential methylation statistics between mutants and the WT, and the "getMethylDiff" function was used to select the DMRs with parameters "difference $=8$, qvalue $=0.01$ ". The selected 500 -bp DMRs were merged using the "merge" command from bedtools ${ }^{47}$. For the PCA analysis (Supplementary Fig. 2b), the methylation levels were calculated in 50-kb tiling window with a 10-kb step size across the genome. The two replicates of WGBS data for nrpd1-3 and nrpe1-11 were downloaded from the NCBI GEO database with accession numbers GSE44209 ${ }^{14}$ and GSE83802 ${ }^{48}$.

\section{Small RNA data analysis}

Total RNA was extracted from 2-week-old seedlings using the TRIzol (Invitrogen) method. RNA samples were separated on a PAGE gel, and the 18- to 30-nt fraction of the gel was cut for small RNA purification. Library preparation and sequencing were performed using Illumina reagents according to the manufacturer's instructions at the Genomics Core Facility of PSC. Small RNA data were analyzed 
according to ${ }^{49}$ with minor modifications. In brief, the adapter was removed using the "fastx_clipper" command from FASTX-toolkit (http://hannonlab.cshl.edu/fastx_toolkit/index.html). After adapter sequences were trimmed, clean reads with sizes ranging from 18- to 31-nt were mapped to the Arabidopsis genome (TAIR10) using Bowtie ${ }^{50}$ with parameters "-v 0 -k 10". Reads that overlapped with annotated tRNAs, rRNAs, snRNAs, or snoRNAs were excluded. Read counts were normalized to Reads Per Ten Million (RPTM) based on the total abundance of genome-matched small RNA reads. The "hitsnormalized-abundance" (HNA) values were calculated by dividing the normalized abundance (in RPTM) for each small RNA hit, where a hit is defined as the number of loci at which a given sequence perfectly matches the genome ${ }^{51}$. The HNA values of small RNAs of all sizes within individual non-overlapping 200-bp windows throughout the whole genome were compared between the mutant and the WT. To focus on regions that show adequate small RNA accumulation in the WT plants, HNA values in the mutant and the WT samples were summed, and a cutoff of 25 was applied. Subsequently, only those 200-bp regions that showed $\geq 2$-fold HNA reduction in the mutant were identified as small RNA-depleted regions. In Fig. 3a, small RNA data for Col-0, rdm15-2, and $r d m 15-3$ were sequenced for this project. Small RNA data for nrpd1 were downloaded from the NCBI GEO database with accession number GSE83802 ${ }^{48}$. In Fig. 3b, all of the small RNA data were downloaded from the NCBI GEO database with accession number GSE45368 15 .

\section{Affinity purification and mass spectrometry}

The RDM15genomic sequence was fused in frame to the $3 x F L A G$ tag and $3 x M Y C$ tag, and the fused sequences were inserted into the pCambia 1305 backbone and were transformed into Arabidopsis plants, respectively. A 5-g quantity of flower tissue was harvested from transgenic plants and ground in liquid nitrogen. The proteins were extracted in $25 \mathrm{~mL}$ of lysis buffer [ $50 \mathrm{mM}$ Tris $(\mathrm{pH} 7.5), 150 \mathrm{mM} \mathrm{NaCl}, 5 \mathrm{mM}$ $\mathrm{MgCl}_{2}, 10 \%$ glycerol, $0.1 \%$ Nonidet P-40, $0.5 \mathrm{mM}$ DTT, $1 \mathrm{mM} \mathrm{PMSF}$, and 250 ul of plant cell protease inhibitor (Sigma)]. After the proteins were extracted, anti-Flag M2 (Sigma; F3165) or anti- Myc (Sigma; M5546) was added and incubated at $4{ }^{\circ} \mathrm{C}$ for $2-3 \mathrm{~h}$. The resins were then washed with the lysis buffer at least five times. The protein samples were sent to the Core Facility for Proteomics at the Shanghai Center for Plant Stress Biology (PSC) for affinity purification and mass spectrometry.

\section{Histone peptide array}

Histone peptide array assay was performed using the MODified Histone Peptide Array kit (Active Motif) according to the manufacturer's instructions. In brief, the slide was first blocked for $2 \mathrm{~h}$ at room

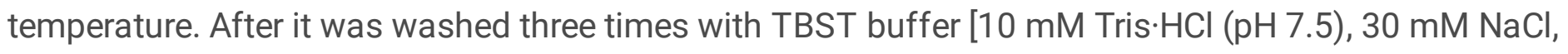
and $0.05 \%$ Tween-20], the slide was incubated overnight with $10 \mathrm{mg}$ of GST-fused purified RDM15 protein at $4{ }^{\circ} \mathrm{C}$ in $10 \mathrm{~mL}$ of binding buffer [50 mM Hepes (pH 8.0), $150 \mathrm{mM} \mathrm{NaCl}, 2 \mathrm{mM}$ DTT, and 0.05\% Nonidet 
P-40]. After it was washed three additional times with TBST buffer, the slide was incubated with anti-GST primary antibody and secondary antibody in TBST buffer for $1 \mathrm{~h}$ at room temperature, respectively. After the washing, the slide was visualized using lumi-light ECL substrate (Roche). The intensity of the blotted slide was analyzed according to the instructions of the analysis software of the MODified Histone Peptide Array.

\section{ChIP-seq analysis}

The ChIP assay was performed as previously described ${ }^{52}$. Protein $\mathrm{G}$ dynabeads (Invitrogen, cat\# 10003D) were used for antibody binding. The antibody for FLAG was anti-Flag M2 (Sigma; F3165).

The quality of the sequencing data was checked with FastQC (http://www.bioinformatics.babraham.ac.uk/projects/fastqc/). The paired-end reads were mapped to the TAIR10 genome of Arabidopsis thaliana (TAIR10) with bowtie2 ${ }^{50}$ with parameter "--very-sensitive --nounal -no-mixed-no-discordant -k 5" . To remove potential PCR duplicates, markdup from SAMtools ${ }^{46}$ was used. After mapping, only uniquely mapped reads were retained for downstream analysis. Fragment number of interested regions was counted by featureCounts ${ }^{53}$ with parameters "-p -0". The RDM15 enrichment (H3K4me1) signal was calculated as

$\left.\log 2\left[\left(1+n \_C h I P\right) / N \_C h I P\right]-\log 2\left[\left(1+n \_I n p u t\right) / N \_I n p u t\right)\right]$, where n_ChIP and n_Input represent the number of mapped ChIP and Input fragments in the interested regions, and N_ChIP and N_Input are the number of all mapped unique fragments. The public data for $\mathrm{H} 3 \mathrm{~K} 4 \mathrm{me} 2$ and $\mathrm{H} 3 \mathrm{~K} 4 \mathrm{me} 3$ were downloaded from the NCBI GEO database with accession number GSE113076.

\section{BiFC}

Agrobacterium tumefaciens strain GV3101 carrying constructs expressing RDM15- cYFP, NRPE3B-nYFP and RDM15- $n Y F P$, or NRPE3B-CYFP were infiltrated into $N$. benthamiana leaves. After $2 \mathrm{~d}$, the infiltrated areas were examined with a Leica TCS-SP8 microscope.

\section{Split luciferase complementation assays}

Split luciferase complementation assays were performed in tobacco leaves. The coding sequences of RDM15 and NRPE3B were cloned into pCAMBIA-nLUC and PCAMBIA-CLUC vectors ${ }^{54}$. A. tumefaciens GV3101 carrying different constructs were infiltrated into N. benthamiana leaves. Two days after infiltration, luciferase activity was detected with a luminescence imaging system (Princeton Instruments) as described by Lang et al. (2015). 


\section{Real-time RT-PCR analysis}

For real-time RT-PCR analysis, total RNA was extracted and contaminating

DNA was removed with RNase-free DNase (RNeasy mini kit; Qiagen). mRNA

(1 mg) was used for the first-strand cDNA synthesis with Takara RT-

PCR Systems following the manufacturer's instructions. The cDNA

reaction mixture was then diluted five times, and $1 \mathrm{ul}$ was used as template in

a 15-ml PCR reaction with SYBR Green mix (Takara). Primer sequence information is listed in Supplementary Table 6.

\section{Protein expression and purification}

The tudor domain of Arabidopsis thaliana RDM15 (residues 598-662) was cloned into a pET-Sumo vector to fuse a hexahistidine and yeast Sumo tag to the target protein. The recombinant protein was expressed in E. coli strain BL21(DE3) by IPTG induction with a concentration of $0.3 \mathrm{mM}$. The protein was purified using a HisTrap (GE Healthcare) column. The His-Sumo tag was removed by ulp1 digestion followed by a second step with HisTrap column. The target protein was further purified using a Superdex G75 column (GE Healthcare). The Se-Met labelled protein was expressed in Se-Met (Anatrace) containing M9 medium and was purified using the same protocol as used for the WT protein. All of the mutants were generated using a PCR-based mutagenesis method and were expressed and purified using the same protocol as used for the WT protein. All peptides used in this research were synthesized by GL Biochem (Shanghai).

\section{Crystallization, data collection, and structure determination}

Before crystallization, the purified RDM15 tudor domain was concentrated to $20 \mathrm{mg} / \mathrm{ml}$ and mixed with $\mathrm{H} 3(1-15) \mathrm{K} 4 \mathrm{me} 1$ peptide with a molar ratio of $1: 4$ at $4{ }^{\circ} \mathrm{C}$ for $30 \mathrm{~min}$. The crystallization was conducted using the sitting-drop vapor diffusion method at $20^{\circ} \mathrm{C}$. The RDM15 tudor domain in complex with H3K4me1 peptide was crystallized in a condition of $0.1 \mathrm{M} \mathrm{Tris-HCl,} \mathrm{pH} 8.5$, and $20 \%$ PEG 1000 . The crystals were soaked in the reservoir solution supplemented with $15 \%$ glycerol and were flash cooled in liquid nitrogen for data collection. All the diffraction data were collected at the Shanghai Synchrotron Radiation Facility beamline BL17U1 and were processed using the HKL2000 program ${ }^{55}$, 56 . The structure of the RDM15 tudor domain in complex with the H3K4me1 peptide was solved using the SAD method implemented in the Phenix program ${ }^{57}$. Model building was conducted 
using the Coot program ${ }^{58}$. The geometry of the model was analyzed using the MolProbity program ${ }^{59}$. All of the molecular graphics were generated using the program Pymol (DeLano Scientific LLC). A summary of diffraction data and structure refinement statistics is provided in Supplementary Table 5.

\section{Isothermal titration calorimetry}

ITC binding was measured using a Microcal PEAQ-ITC instrument (Malvern). The purified proteins were dialyzed against a buffer containing $100 \mathrm{mM} \mathrm{NaCl}, 20 \mathrm{mM} \mathrm{HEPES}, \mathrm{pH} 7.0$, and $2 \mathrm{mM}$ b-mercaptoethanol. The peptide was dissolved in the same buffer. All of the titration was performed at $20^{\circ} \mathrm{C}$. The data were analyzed using the Origin 7.0 program.

\section{Accession codes}

The data discussed in this publication have been deposited in NCBI's Gene Expression Omnibus ${ }^{60}$ and are accessible through GEO Series accession number GSE154302. The structure of the Arabidopsis RDM15 tudor domain in complex with an H3K4me1 peptide has been deposited into the RCSB Protein Data Bank with the accession code of XXXX.

\section{Declarations}

\section{Acknowledgements}

We thank the staff at beamline BL17U1 at the Shanghai Synchrotron Radiation Facility for their help with data collection. This work was supported by the National Key R\&D Program of China (2018YFD1000200 to Z.L., 2016 YFA0503200 to J.D.), the Strategic Priority Research Program of the Chinese Academy of Sciences (XDB27040000 to Z. L., XDB27040101 to J.-K.Z.), Shenzhen Science and Technology Program (KQTD20190929173906742 to J.D.), SUSTech (G02226301 to J.D.), and the Key Laboratory of Molecular Design for Plant Cell Factory of Guangdong Higher Education Institutes (2019KSYS006 to J.D.).

\section{References}

1. Zhang, H., Lang, Z. \& Zhu, J.K. Dynamics and function of DNA methylation in plants. Nat Rev Mol Cell Biol 19, 489-506 (2018).

2. He, X.J., Chen, T. \& Zhu, J.K. Regulation and function of DNA methylation in plants and animals. Cell Res 21, 442-465 (2011).

3. Finnegan, E.J., Peacock, W.J. \& Dennis, E.S. Reduced DNA methylation in Arabidopsis thaliana results in abnormal plant development. Proc Natl Acad Sci U S A 93, 8449-8454 (1996). 
4. Lindroth, A.M. et al. Requirement of CHROMOMETHYLASE3 for maintenance of CpXpG methylation. Science 292, 2077-2080 (2001).

5. Cao, X. \& Jacobsen, S.E. Locus-specific control of asymmetric and $\mathrm{CpNpG}$ methylation by the DRM and CMT3 methyltransferase genes. Proc Natl Acad Sci U S A 99 Suppl 4, 16491-16498 (2002).

6. Zemach, A. et al. The Arabidopsis nucleosome remodeler DDM1 allows DNA methyltransferases to access H1-containing heterochromatin. Cell 153, 193-205 (2013).

7. Liu, R. \& Lang, Z. The mechanism and function of active DNA demethylation in plants. J Integr Plant Biol 62, 148-159 (2020).

8. Zhang, H. \& Zhu, J.K. RNA-directed DNA methylation. Curr Opin Plant Biol 14, 142-147 (2011).

9. Matzke, M.A. \& Mosher, R.A. RNA-directed DNA methylation: an epigenetic pathway of increasing complexity. Nat Rev Genet 15, 394-408 (2014).

10. Ali, M. et al. RNA-directed DNA methylation in plants. 34, 1857-1862 (2015).

11. Du, J., Johnson, L.M., Jacobsen, S.E. \& Patel, D.J. DNA methylation pathways and their crosstalk with histone methylation. Nat Rev Mol Cell Biol 16, 519-532 (2015).

12. Liu, R., Li, X., Chen, W. \& Du, J. Structure and mechanism of plant histone mark readers. Sci China Life Sci 61, 170-177 (2018).

13. Zhao, S., Zhang, B., Yang, M., Zhu, J. \& Li, H. Systematic Profiling of Histone Readers in Arabidopsis thaliana. Cell Rep 22, 1090-1102 (2018).

14. Zhang, H. et al. DTF1 is a core component of RNA-directed DNA methylation and may assist in the recruitment of Pol IV. Proc Natl Acad Sci U S A 110, 8290-8295 (2013).

15. Law, J.A. et al. Polymerase IV occupancy at RNA-directed DNA methylation sites requires SHH1. Nature 498, 385-389 (2013).

16. Greenberg, M.V.C. et al. Interplay between Active Chromatin Marks and RNA-Directed DNA Methylation in Arabidopsis thaliana. 9, e1003946 (2013).

17. Liu, Z.W. et al. The SET domain proteins SUVH2 and SUVH9 are required for Pol V occupancy at RNAdirected DNA methylation loci. PLoS Genet 10, e1003948 (2014).

18. Johnson, L.M., Law, J.A., Khattar, A., Henderson, I.R. \& Jacobsen, S.E. SRA-domain proteins required for DRM2-mediated de novo DNA methylation. PLoS Genet 4, e1000280 (2008).

19. Johnson, L.M. et al. SRA- and SET-domain-containing proteins link RNA polymerase $V$ occupancy to DNA methylation. 507, 124 (2014).

20. Gong, Z. et al. ROS1, a repressor of transcriptional gene silencing in Arabidopsis, encodes a DNA glycosylase/lyase. Cell 111, 803-814 (2002).

21. Gao, Z. et al. An RNA polymerase II- and AGO4-associated protein acts in RNA-directed DNA methylation. Nature 465, 106-109 (2010).

22. He, X.J. et al. A conserved transcriptional regulator is required for RNA-directed DNA methylation and plant development. Genes Dev 23, 2717-2722 (2009). 
23. He, X.J. et al. An effector of RNA-directed DNA methylation in arabidopsis is an ARGONAUTE 4- and RNA-binding protein. Cell 137, 498-508 (2009).

24. Lei, M. et al. Regulatory link between DNA methylation and active demethylation in Arabidopsis. Proc Natl Acad Sci U S A 112, 3553-3557 (2015).

25. Williams, B.P., Pignatta, D., Henikoff, S. \& Gehring, M. Methylation-sensitive expression of a DNA demethylase gene serves as an epigenetic rheostat. PLoS Genet 11, e1005142 (2015).

26. Akalin, A. et al. methylKit: a comprehensive R package for the analysis of genome-wide DNA methylation profiles. Genome Bio/ 13, R87 (2012).

27. Lu, R. \& Wang, G.G. Tudor: a versatile family of histone methylation 'readers'. Trends Biochem Sci 38, 546-555 (2013).

28. Patel, D.J. \& Wang, Z. Readout of epigenetic modifications. Annu Rev Biochem 82, 81-118 (2013).

29. Zhang, K., Sridhar, V.V., Zhu, J., Kapoor, A. \& Zhu, J.K. Distinctive core histone post-translational modification patterns in Arabidopsis thaliana. PLoS One 2, e1210 (2007).

30. Patel, D.J. A Structural Perspective on Readout of Epigenetic Histone and DNA Methylation Marks. Cold Spring Harb Perspect Biol 8, a018754 (2016).

31. Liu, Q. \& Gong, Z. The coupling of epigenome replication with DNA replication. Curr Opin Plant Biol 14, 187-194 (2011).

32. Zheng, X. et al. ROS3 is an RNA-binding protein required for DNA demethylation in Arabidopsis. Nature 455, 1259-1262 (2008).

33. Cao, X. et al. Role of the DRM and CMT3 methyltransferases in RNA-directed DNA methylation. Curr Biol 13, 2212-2217 (2003).

34. Dinh, T.T. et al. Generation of a luciferase-based reporter for CHH and CG DNA methylation inArabidopsis thaliana. Silence 4, 1 (2013).

35. Gupta, J., Kumar, S., Li, J., Krishna Murthy Karuturi, R. \& Tikoo, K. Histone H3 lysine 4 monomethylation (H3K4me1) and H3 lysine 9 monomethylation (H3K9me1): distribution and their association in regulating gene expression under hyperglycaemic/hyperinsulinemic conditions in 3T3 cells. Biochimie 94, 2656-2664 (2012).

36. Herz, H.M. et al. Enhancer-associated H3K4 monomethylation by Trithorax-related, the Drosophila homolog of mammalian MII3/MII4. Genes Dev 26, 2604-2620 (2012).

37. Li, H. et al. Structural basis for lower lysine methylation state-specific readout by MBT repeats of L3MBTL1 and an engineered PHD finger. Mol Cel/ 28, 677-691 (2007).

38. Min, J. et al. L3MBTL1 recognition of mono- and dimethylated histones. Nat Struct Mol Biol 14, 1229-1230 (2007).

39. Liu, Y. \& Huang, Y. Uncovering the mechanistic basis for specific recognition of monomethylated H3K4 by the CW domain of Arabidopsis histone methyltransferase SDG8. J Biol Chem 293, 64706481 (2018). 
40. Zhao, S. et al. Kinetic and high-throughput profiling of epigenetic interactions by 3D-carbene chipbased surface plasmon resonance imaging technology. Proc Natl Acad Sci U S A 114, E7245-E7254 (2017).

41. Cai, L. et al. An H3K36 methylation-engaging Tudor motif of polycomb-like proteins mediates PRC2 complex targeting. Mol Cell 49, 571-582 (2013).

42. Ballare, C. et al. Phf19 links methylated Lys 36 of histone $\mathrm{H} 3$ to regulation of Polycomb activity. Nat Struct Mol Biol 19, 1257-1265 (2012).

43. Musselman, C.A. et al. Molecular basis for H3K36me3 recognition by the Tudor domain of PHF1. Nat Struct Mol Biol 19, 1266-1272 (2012).

44. Bolger, A.M., Lohse, M. \& Usadel, B. Trimmomatic: a flexible trimmer for Illumina sequence data. Bioinformatics 30, 2114-2120 (2014).

45. Xi, Y. \& Li, W. BSMAP: whole genome bisulfite sequence MAPping program. BMC Bioinformatics 10, 232 (2009).

46. Li, H. et al. The Sequence Alignment/Map format and SAMtools. Bioinformatics 25, 2078-2079 (2009).

47. Quinlan, A.R. \& Hall, I.M. BEDTools: a flexible suite of utilities for comparing genomic features. Bioinformatics 26, 841-842 (2010).

48. Tang, K., Lang, Z., Zhang, H. \& Zhu, J.K. The DNA demethylase ROS1 targets genomic regions with distinct chromatin modifications. Nat Plants 2, 16169 (2016).

49. Zhang, H. et al. An Rrp6-like protein positively regulates noncoding RNA levels and DNA methylation in Arabidopsis. Mol Cell 54, 418-430 (2014).

50. Langmead, B. \& Salzberg, S.L. Fast gapped-read alignment with Bowtie 2. Nat Methods 9, 357-359 (2012).

51. Lee, T.F. et al. RNA polymerase V-dependent small RNAs in Arabidopsis originate from small, intergenic loci including most SINE repeats. Epigenetics 7, 781-795 (2012).

52. Wierzbicki, A.T., Haag, J.R. \& Pikaard, C.S. Noncoding transcription by RNA polymerase Pol IVb/Pol V mediates transcriptional silencing of overlapping and adjacent genes. Cell 135, 635-648 (2008).

53. Liao, Y., Smyth, G.K. \& Shi, W. featureCounts: an efficient general purpose program for assigning sequence reads to genomic features. Bioinformatics 30, 923-930 (2014).

54. Chen, H. et al. Firefly luciferase complementation imaging assay for protein-protein interactions in plants. Plant Physiol 146, 368-376 (2008).

55. Minor, W., Cymborowski, M., Otwinowski, Z. \& Chruszcz, M. HKL-3000: the integration of data reduction and structure solution-from diffraction images to an initial model in minutes. Acta Crystallogr D Biol Crystallogr 62, 859-866 (2006).

56. Wang, Q.-S. et al. Upgrade of macromolecular crystallography beamline BL17U1 at SSRF. Nuclear Science and Techniques 29, 68 (2018). 
57. Adams, P.D. et al. PHENIX: a comprehensive Python-based system for macromolecular structure solution. Acta Crystallogr D Biol Crystallogr 66, 213-221 (2010).

58. Emsley, P., Lohkamp, B., Scott, W.G. \& Cowtan, K. Features and development of Coot. Acta Crystallogr D Biol Crystallogr 66, 486-501 (2010).

59. Chen, V.B. et al. MolProbity: all-atom structure validation for macromolecular crystallography. Acta Crystallogr D Biol Crystallogr 66, 12-21 (2010).

60. Edgar, R., Domrachev, M. \& Lash, A.E. Gene Expression Omnibus: NCBI gene expression and hybridization array data repository. Nucleic Acids Res 30, 207-210 (2002).

\section{Figures}
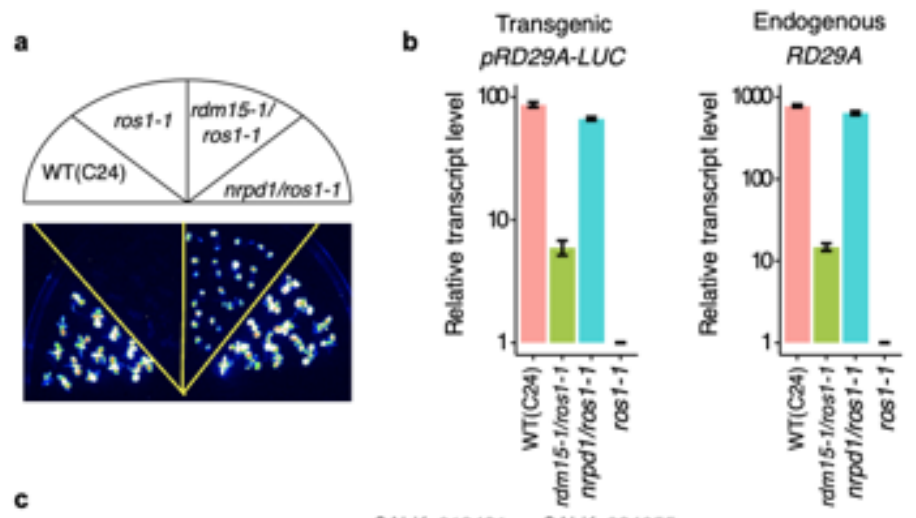

c

SALK_013481 SALK_024055

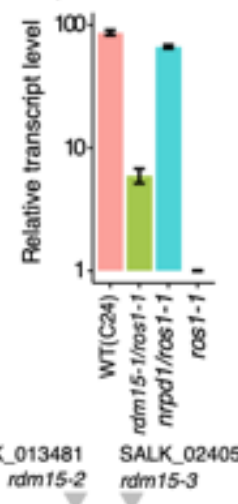

AT 4 G31880

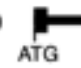

ram15.2 ram15-3
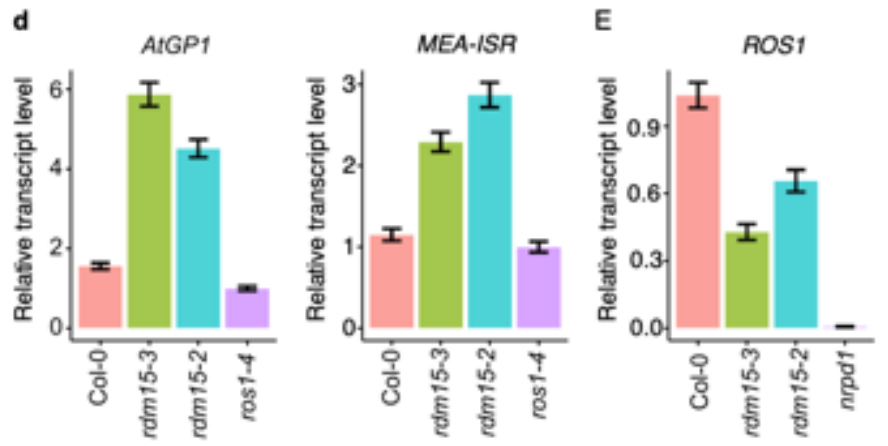

\section{Figure 1}

RDM15 mediates transcriptional silencing. a. Isolation of the rdm15-1 mutant. The wild-type (WT) of the C24 ecotype carries a stress-inducible RD29A-LUC transgene, the expression of which can be assessed by luminescence. The WT, ros1-1, rdm15-1 ros1-1, and nrpd1 ros1-1 plants were grown for 10 days and imaged after cold treatment $\left(48 \mathrm{~h}, 4^{\circ} \mathrm{C}\right)$. b. RT-qPCR analysis of relative transcript levels of transgenic RD29A-LUC and endogenous RD29A in the WT, rdm15-1 ros1-1, nrpd1 ros1-1, and ros1-1. Ten-day-old seedlings were used for RNA extraction after cold treatment $\left(48 \mathrm{~h}, 4^{\circ} \mathrm{C}\right)$. c. Diagram showing the positions 
of T-DNA insertions in rdm15-1, rdm15-2, and rdm15-3. Boxes and lines denote exons and introns of RDM15 (AT4G31880), respectively. d. RT-qPCR analysis of relative transcript levels of AtGP1 and MEA_ISR in Col-0 (WT), rdm15-2, rdm15-3, and ros1-4. e. RT-qPCR analysis of relative transcript level of ROS1 in Col-0, rdm15-2, rdm15-3, and nrpd1-3. ACTIN2 served as the internal control in the RT-qPCR analysis. Error bars represent s.d. $(n=3)$.
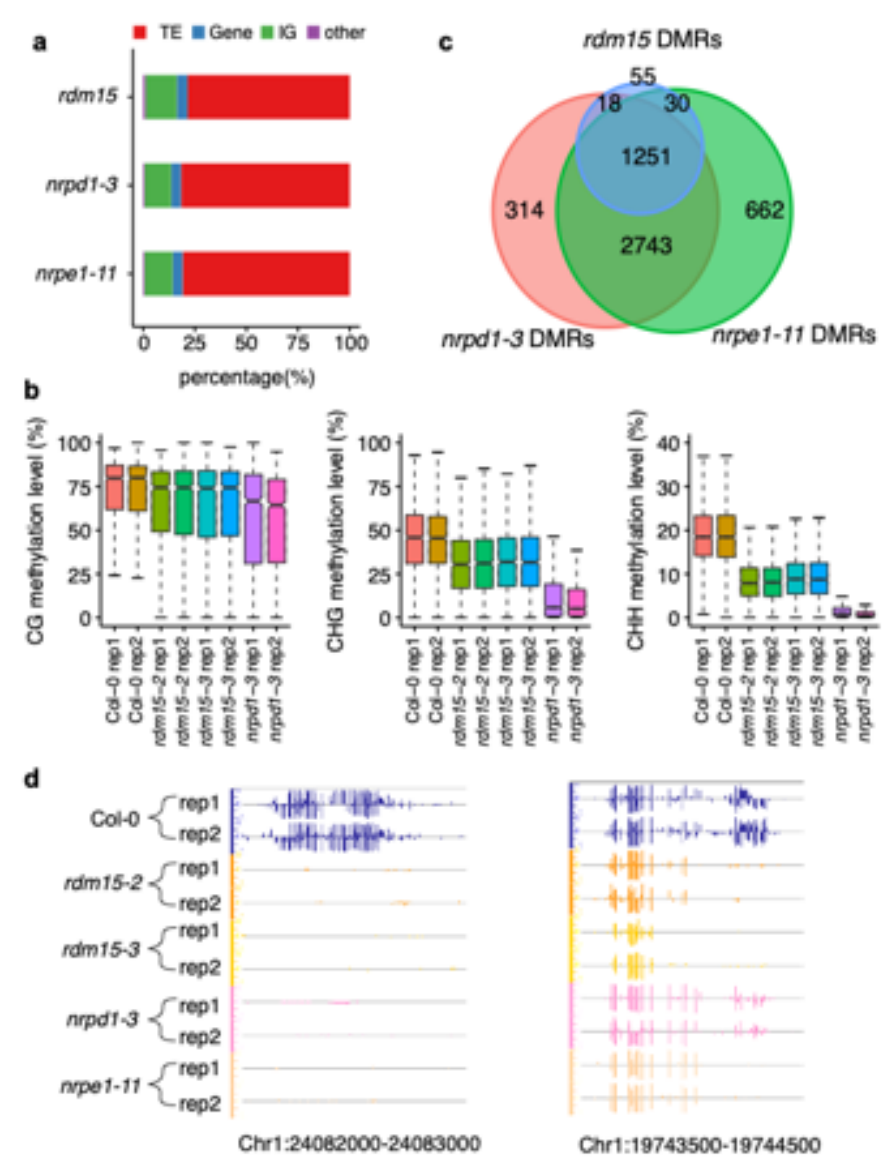

\section{Figure 2}

RDM15 is required for RdDM-dependent DNA methylation. a. Compositions of DMRs among TEs, genes, and intergenic regions (IGs). DMRs (relative to the WT) were identified in rdm15, nrpd1-3, and nrpe1-11. b. Venn diagram showing the numbers of DMRs that overlapped between rdm15, nrpd1-3, and nrpe1-11. C. Box plots showing CG, CHG, and CHH methylation levels of rdm15 DMRs in Col-0, rdm15-2, rdm15-3, and nrpd1-3. Data from two replicates are shown. d. The Integrative Genome Browser (IGB) display of wholegenome bisulfite sequencing (WGBS) data is shown for several representative rdm15 DMRs in Col-0, rdm15-2, rdm15-3, and nrpd1-3. Vertical bars on each track indicate DNA methylation levels. The coordinates of DMRs are indicated below the snapshots. Two replicates are shown for each genotype. 
a
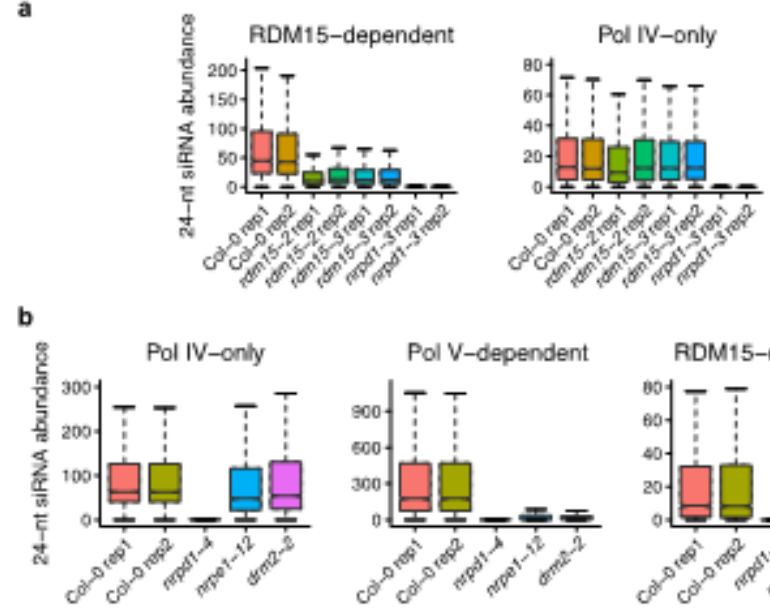

c

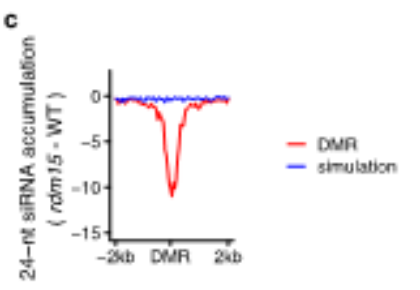

d
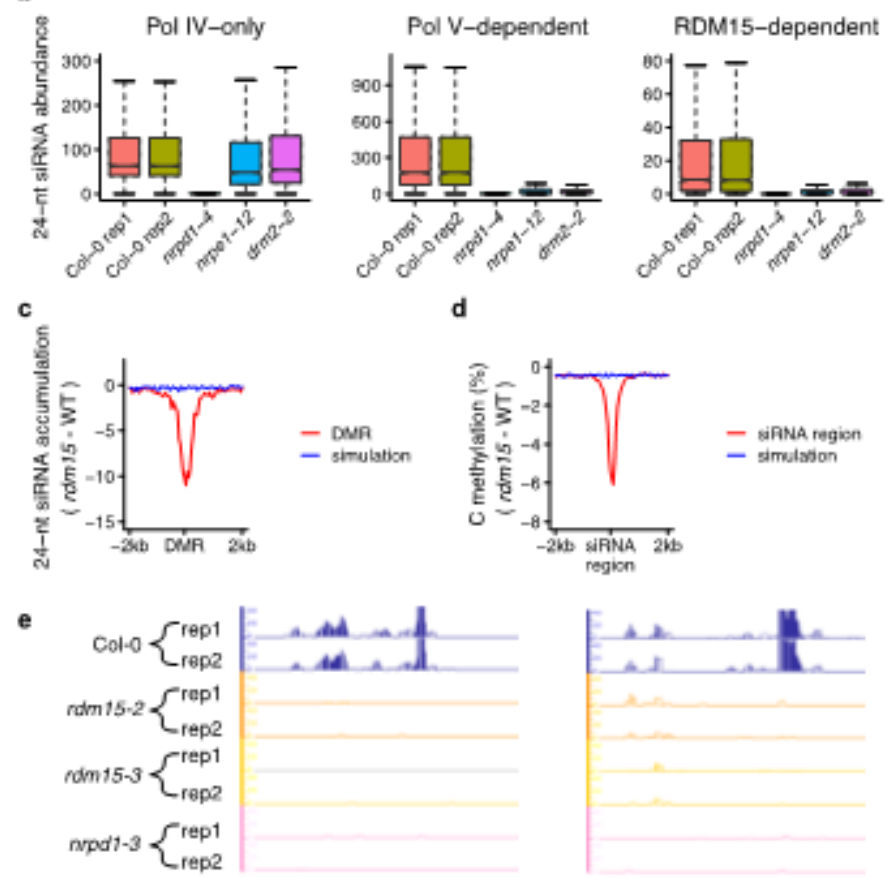

Chr1:24082000-24083000

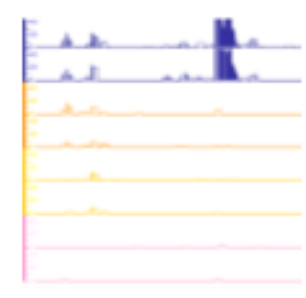

Chr1:19743500-19744500

\section{Figure 3}

Dysfunction of RDM15 abolishes RdDM-dependent 24-nt siRNA accumulation. a. Boxplots showing the abundance of 24-nt siRNAs in Col-0, rdm15-2, rdm15-3, and nrpd1-3 with two replicates. The siRNA levels of RDM15-dependent siRNA cluster regions (left) and Pol IV-only siRNA cluster regions (right) are shown. Compared to Col-0, RDM15 siRNAs were decreased in rdm15 and nrpd1-3 mutants, whereas Pol IV-only siRNAs (upsteam RdDM siRNAs) were decreased in nrpd1-3 but not in rdm15. b. The abundance of 24-nt siRNAs is shown for Pol IV-only, Pol V-dependent, and RDM15-dependent siRNA regions in Col-0, nrpd1-4, nrpe1-12, and drm2-2. Pol IV-only and Pol V-dependent siRNAs represent upstream and downstream RdDM siRNAs, respectively. c. Changes in 24-nt siRNA enrichment in rdm15 relative to WT at rdm15 hypo DMRs. d. Changes in DNA methylation level, including $\mathrm{mCG}, \mathrm{mCHG}$, and $\mathrm{mCHH}$, in rdm15 relative to the WT at RDM15-dependent siRNA cluster regions. E. IGB display of siRNA abundance of two RDM15dependent siRNA regions in Col-0, rdm15-2, rdm15-3, and nrpd1-3. Two replicates are shown. 


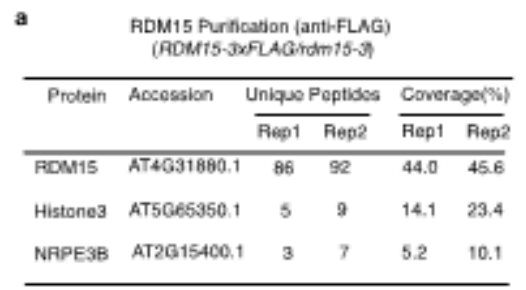

\begin{tabular}{|c|c|c|c|c|c|}
\hline \multirow[t]{2}{*}{ Protein } & \multirow[t]{2}{*}{ Acoossion } & \multicolumn{2}{|c|}{ Unique Psptides } & \multicolumn{2}{|c|}{ Coverage(S) } \\
\hline & & Rep1 & Aep2 & Rep1 & Rep? \\
\hline RDM15 & AT $4 \mathrm{G} 31880$. & 156 & 74 & 36.8 & 42.9 \\
\hline Histonea & ATSG65350. & 13 & 5 & 10.4 & 14.1 \\
\hline NRPE3B & AT2615400 & 2.15 & 4 & 8.4 & 6.7 \\
\hline
\end{tabular}

b
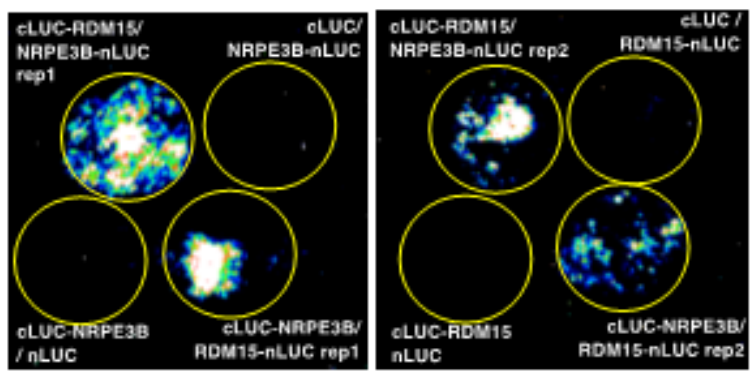

c

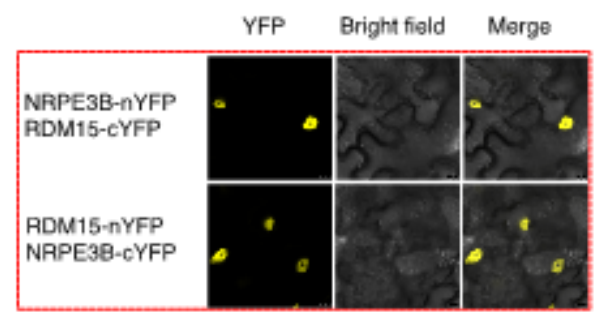

\section{Figure 4}

RDM15 physically interacts with NRPE3B, a subunit exclusive to Pol V. a. Detection of proteins associated with RDM15. LC-MS/MS was performed following immunoprecipitation of FLAG-tagged RDM15 and MYC-tagged RDM15. There were two biological replicates for each of the protein purifications. $b$. Tests of RDM15-NRPE3B interaction by split luciferase complementation assay in tobacco leaves. Circles indicate leaf regions that were infiltrated with Agrobacterium strains containing the indicated constructs. $C$. Analyses of RDM15-NRPE3B protein interaction by bimolecular fluorescent complimentation assay in tobacco leaves. Yellow fluorescence indicates positive protein interaction.
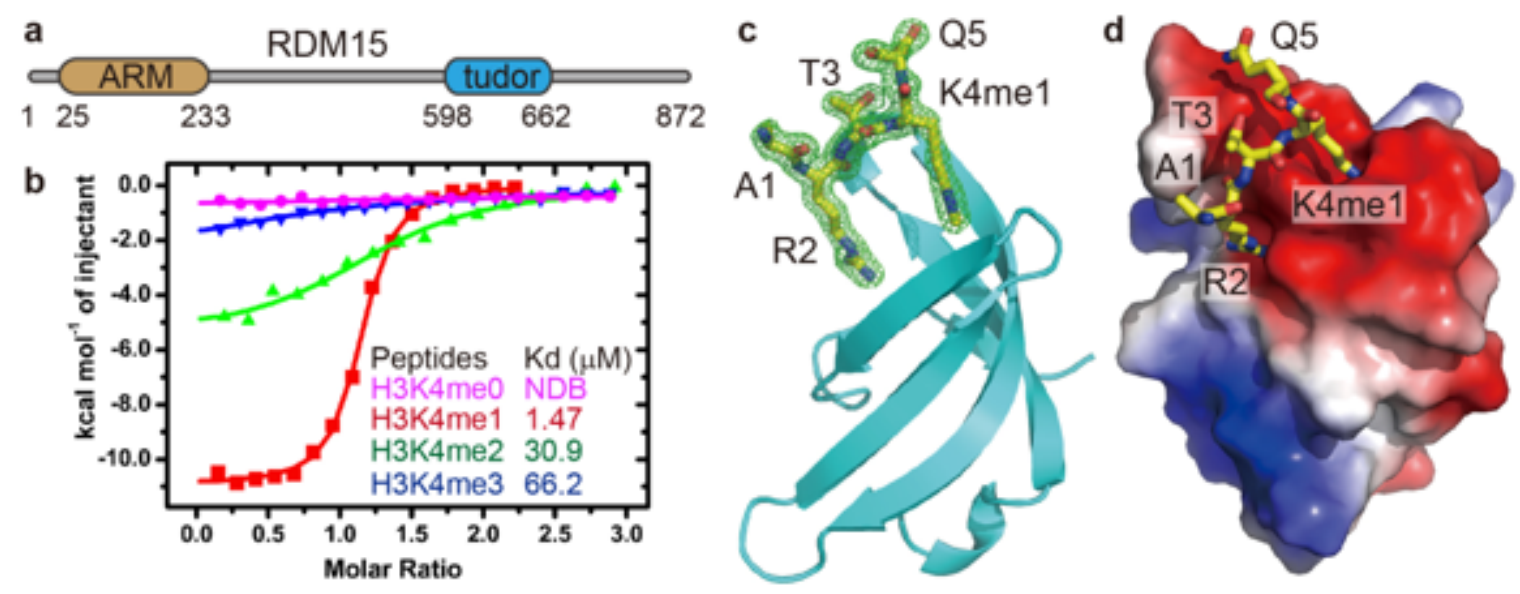

Figure 5 
The RDM15 tudor domain specifically recognizes H3K4me1 and the structure of its complex with the H3K4me1 peptide. a. Schematic representation of the domain architecture of RDM15. b. ITC binding curves between the RDM1 5 tudor domain and peptides with different methylation states on the H3K4 site; the curves indicate that RDM15 prefers the H3K4me1 mark over either lower (H3K4me0) or higher (H3K4me2 or H3K4me3) methylation states. NDB, no detectable binding. c. Overall structure of the RDM15 tudor domain in complex with the H3K4me1 peptide, with RDM15 shown as cyan ribbon and the peptide shown as stick representation. The composite-omit electrondensity map at $1 \otimes$ level of the bound peptide is shown in a green mesh. $d$. The RDM15 tudor-H3K4me1 complex with RDM15 shown in an electrostatics surface and the peptide as stick representation. The interaction is mainly mediated by H3R2 and H3K4me1 residues, which insert their side chains in two negatively charged surface grooves of the tudor domain.

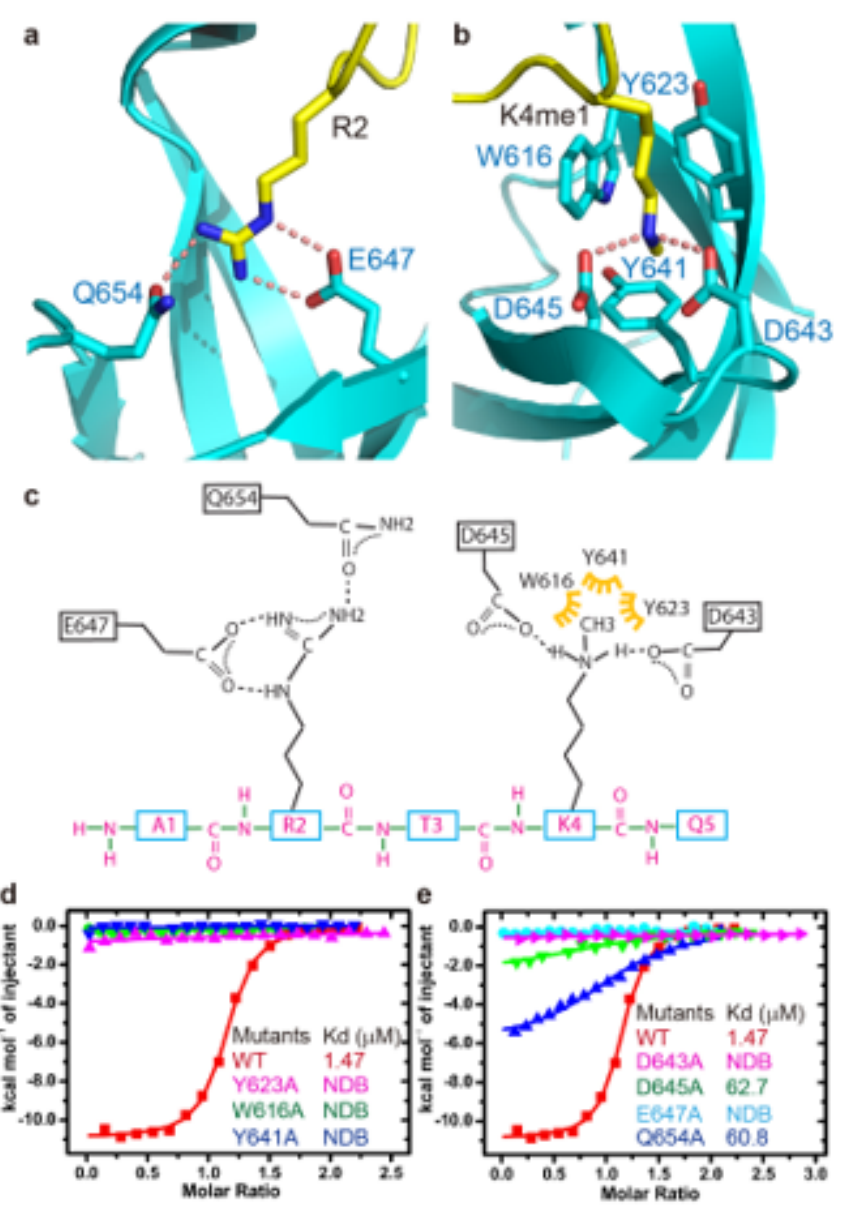

\section{Figure 6}

Details of the specific interaction between the RDM15 tudor domain and the H3K4me1 peptide. a. The side chain of H3R2 is specifically recognized by Glu647 and Gln654 of the tudor domain through salt bridge and hydrogen-bonding interactions. The hydrogen bonds are highlighted with dashed red lines. b. The methyl group of H3K4me1 is specifically accommodated within an aromatic cage formed by Trp616, Tyr623, and Tyr641. The two monomethylammonium protons of monomethyllysine form hydrogen bonding and salt bridge interactions with two negatively charged Asp643 and Asp645 residues. c. A 
schematic representation of the intermolecular interactions between RDM15 and the H3K4me1 peptide. d-e. The ITC binding curves between the H3K4me1 peptide and the RDM15 tudor domain mutations showing that the disruption of the aromatic cage (d) or the residues involved in hydrogen bonding interactions (e) dramatically decreases the binding affinity.
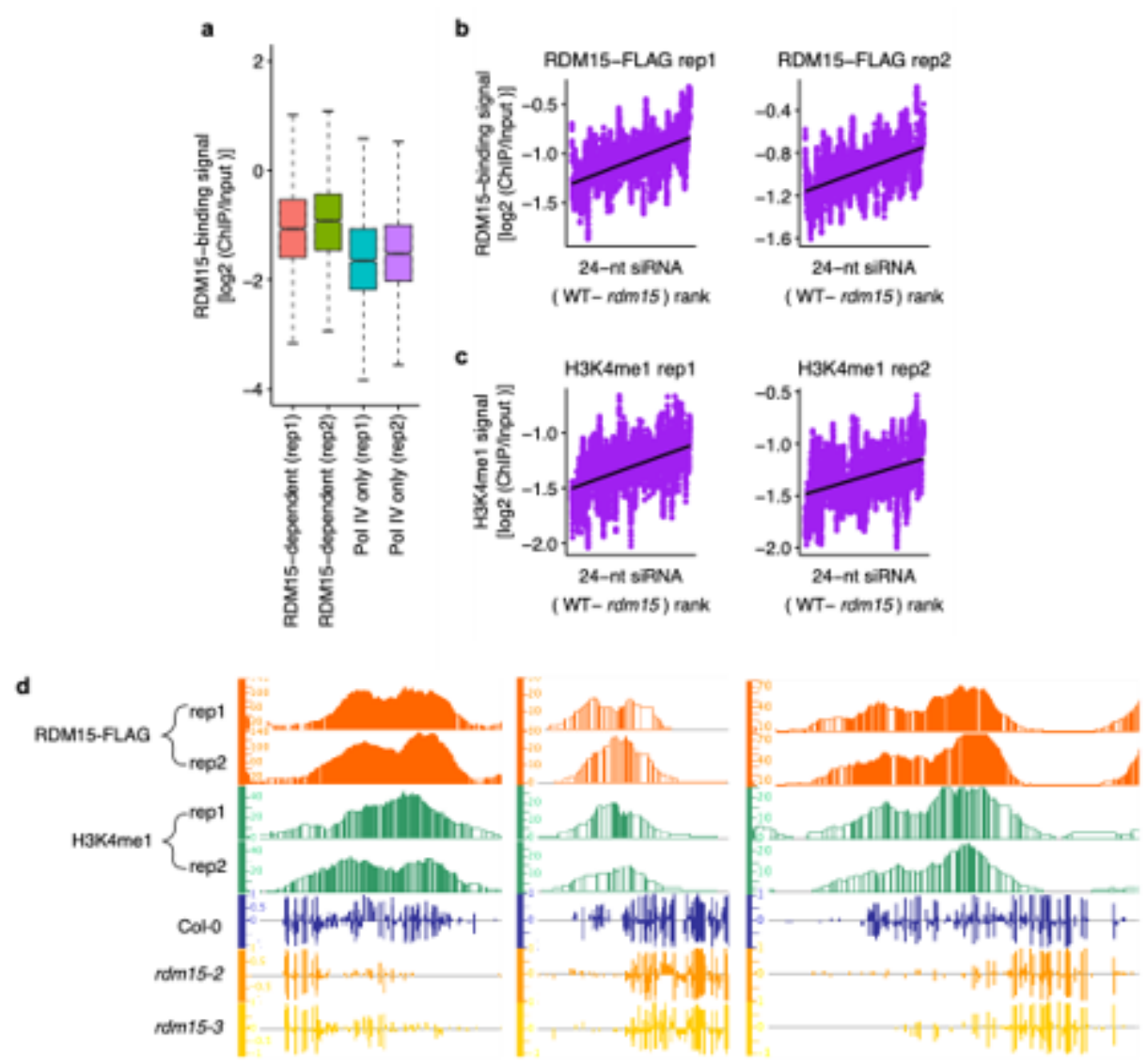

Chr4:7519000-7521000

Chr4-6067000-6068000

Chr1:16413000-16415000

\section{Figure 7}

RDM15 binds to RDM15-dependent siRNA regions in the genome. a. Boxplots showing RDM15 protein enrichment in RDM15-dependent siRNA regions. Pol IV-only siRNA regions, which were not affected in rdm15, served as the control. b. Correlation of the change of siRNA accumulation (WT vs. rdm15) with RDM15 enrichment. RDM15-dependent siRNA regions were ranked by the change of siRNAs (WT vs. rdm15). c. Correlation of the change of siRNA accumulation (WT vs. rdm15) with H3K4me1 enrichment. RDM15-dependent siRNA regions were ranked by the change of siRNAs (WT vs. rdm15). d. IGB display of RDM15 and H3K4me1 protein enrichment and DNA methylation levels at several RDM15 binding targets.

\section{Supplementary Files}

This is a list of supplementary files associated with this preprint. Click to download.

- TableS1DMRlist.xlsx 
- TableS2rdm15siRNAlist.xlsx

- Tables3RDM15PeptideArray.xls

- TableS4RDM15IPMS.xIsx

- SupplementaryTablesandFigures.docx

- RDM15validationreport.pdf 